\title{
Aspetti dermatologici nella malattia di Anderson-Fabry
}

\author{
Giuseppe Pistone, Rosario Gurreri, Maria Rita Bongiorno \\ UOC Dermatologia e MTS, Azienda Ospedaliera Universitaria Policlinico "Paolo Giaccone", Palermo
}

\begin{abstract}
Dermatological manifestations of Anderson-Fabry disease
Dermatological manifestations are an important feature of Fabry disease and include angiokeratomas, telangiectases, lymphedema, anhidrosis or hypohidrosis, and a pseudo-acromegalic facial appearance. Recognition of these symptoms is vital for the early diagnosis and treatment of Fabry disease. It is therefore important that dermatologists as well as other specialists are aware of these manifestations. The currently used causal treatment for Fabry disease is enzyme replacement therapy. Dermatologists play a key role, since cutaneous manifestations may lead to the diagnosis. This may aid in early therapeutic intervention, reducing both morbidity and mortality. Keywords: Angiokeratoma, Anhidrosis, Fabry disease, Hypohidrosis, Lymphedema, Pseudo-acromegalic facial appearance
\end{abstract}

\section{Introduzione}

Nella cute la malattia di Anderson-Fabry (mAF) colpisce principalmente le cellule endoteliali del derma superficiale, dei vasa nervorum nonché le cellule connettivali del perinervio. Inoltre, il globotriaosilceramide (Gb3) può accumularsi nei lisosomi dei periciti vascolari, nelle cellule delle ghiandole eccrine e nei fibroblasti dermici (1). Circa il 78\% dei maschi e il $50 \%$ delle femmine presentano una o più manifestazioni dermatologiche (2).

\section{Angiocheratomi}

Una delle più precoci manifestazioni cliniche della malattia di Anderson-Fabry, riscontrata nel 66\% dei maschi e nel $36 \%$ delle femmine, è rappresentata dagli angiocheratomi (ANC) (3). Costituiscono la più frequente e visibile manifestazione della mAF e, sebbene, possano essere presenti in altre patologie metaboliche e non, vengono considerati un marcatore cutaneo che induce a sospettare la mAF. Si tratta di piccole lesioni papulose, di colorito variabile, dal rosso-

Accepted: March 27, 2017

Published online: August 3, 2017

Indirizzo per la corrispondenza:

Prof. Giuseppe Pistone

UOC Dermatologia e MTS

Azienda Ospedaliera Universitaria

Policlinico "Paolo Giaccone"

Via Del Vespro, 131

90127 Palermo

giuseppe.pistone@unipa.it bruno al rosso-bluastro, della grandezza di qualche millimetro, a volte confluenti (Fig. 1). Di solito, gli angiocheratomi compaiono lentamente durante la prima infanzia e, solo eccezionalmente, possono essere presenti alla nascita. Con il passare del tempo aumentano di numero e di dimensioni. Le lesioni possono essere piane, leggermente rilevate e non scompaiono alla digitopressione, mentre gli elementi di maggiori dimensioni possono essere a superficie ipercheratosica e ruvidi al tatto (4). Nei pazienti maschi, si localizzano elettivamente e simmetricamente nelle regioni glutee, sullo scroto, sul pene, nelle regioni inguinali, nella faccia interna delle cosce, con caratteristica distribuzione "a costume da bagno", raggiungendo, talora, la regione ombelicale dove gli elementi si dispongono a rosetta (5) (Fig. 2). Sono stati descritti casi con interessamento della superficie posteriore delle orecchie e gli angiocheratomi possono localizzarsi anche su altre regioni cutanee quali gomiti, avambracci, dorso delle dita, zone periungueali, torace, collo e labbra. Tali lesioni si possono altresì osservare nelle mucose quali congiuntiva, mucosa orale, delle vie respiratorie, del tratto gastroenterico e genito-urinario. Eterogeneità clinica si ritrova nel genere femminile; talvolta il quadro clinico cutaneo risulta sovrapponibile al genere maschile, in altri casi ritroviamo gli angiocheratomi solo sulla parte superiore del tronco e raramente ai genitali $(6,7)$.

\section{Caratteristiche dermoscopiche e istopatologiche}

La dermatoscopia è una metodica poco costosa e non invasiva che permette la visualizzazione di aspetti morfologici non visibili a occhio nudo. L'angiocheratoma presenta un pattern globale lacunare, con lacune di colore rosso o blu-nerastro, e chiazze nerastre nettamente demarcate, di 


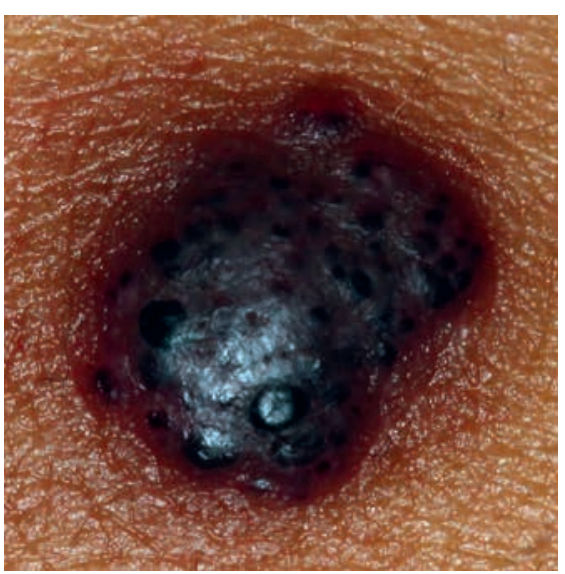

Fig. 1 - Angiocheratoma.

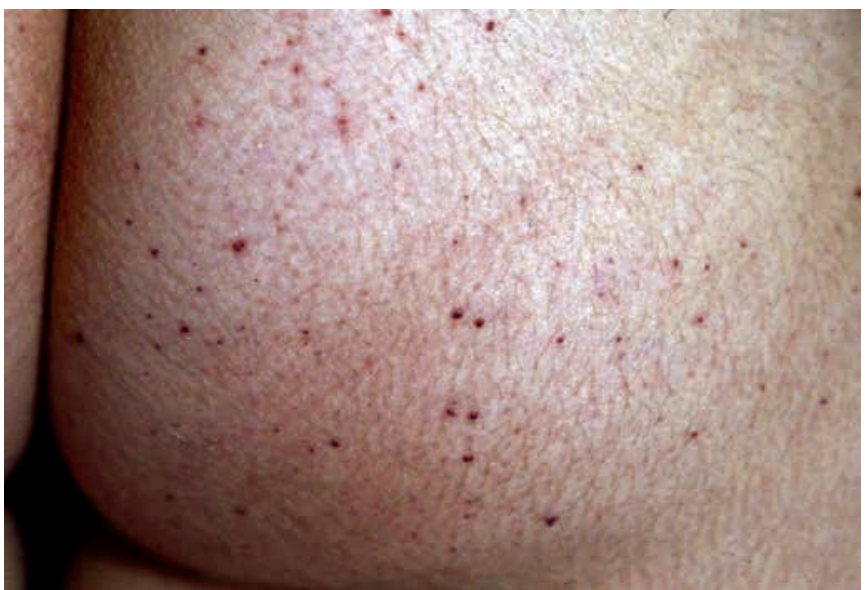

Fig. 2 - Angiocheratomi multipli al gluteo destro.

forma irregolarmente tondeggiante ed estremamente superficiali, corrispondenti a croste ematiche. È possibile rilevare formazioni squamo-crostose, spesse, che appaiono dermoscopicamente come aree opache giallastre, un velo biancastro, mentre in periferia sono spesso presenti una rima traslucida, di aspetto gelatinoso, dovuta a fenomeni di acantosi, e un alone rossastro, conseguente a fenomeni traumatici (8).

Il processo di accumulo patologico nelle cellule e nei tessuti che caratterizza la mAF è facilmente dimostrabile con la biopsia cutanea che permette di documentare gli accumuli glicosfingolipidici elettron-densi al microscopio elettronico e, già al microscopio ottico, evidenzia il sovvertimento dell'organo cute e del microcircolo con ectasie e trombosi diffuse (9). In particolare, all'esame istologico si rileva dilatazione dei vasi superficiali del derma e ipercheratosi dello strato corneo di vario grado (Fig. 3). La dilatazione consegue allo sfiancamento, a causa del deposito di Gb3, della parete vasale, non più adatta a svolgere la sua funzione di vasodilatazione/vasocostrizione. I meccanismi dell'ipercheratosi non sono noti, ma potrebbero essere la conseguenza del-

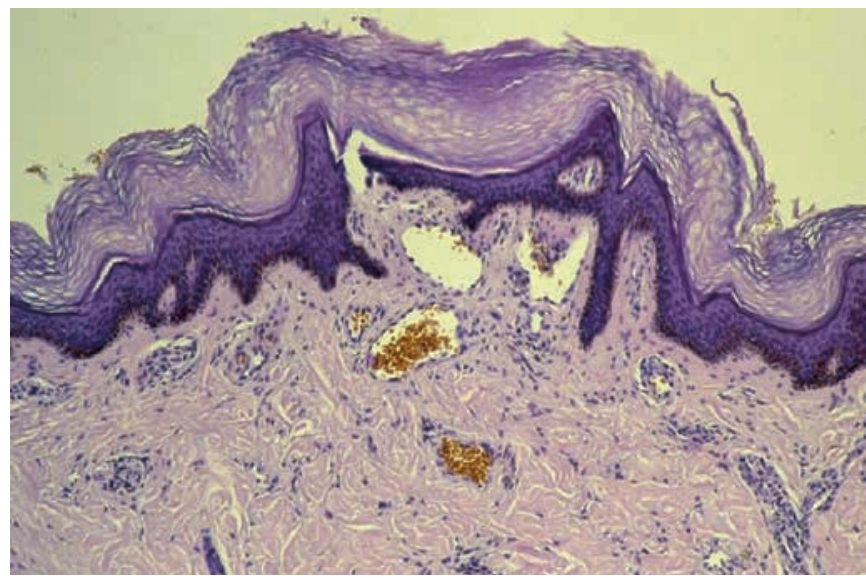

Fig. 3 - Ematossilina-eosina: ipercheratosi e dilatazione dei vasi superficiali del derma.

la pressione esercitata sull'epitelio dai vasi dilatati. Inoltre, sono presenti acantosi con allungamento delle creste interpapillari e dilatazione dei vasi più profondi con formazioni aneurismatiche. I glicosfingolipidi accumulatisi risultano birifrangenti e, se visti mediante luce polarizzata, mostrano una configurazione a "croce di Malta".

\section{Diagnosi differenziale e terapia}

È importante notare che gli angiocheratomi non sono patognomonici della malattia di Anderson-Fabry, infatti si possono osservare in molte altre condizioni patologiche e non, che devono essere ben differenziate. Gli angiocheratomi solitari sono lesioni cutanee comuni, rappresentano il $70 \%$ di tutti gli ANC, spesso di maggiori dimensioni, di colore rosso-nerastro, a superficie liscia o ipercheratosica, entrano in diagnosi differenziale con il melanoma cutaneo (10).

Gli angiocheratomi di Fordyce si evidenziano soprattutto allo scroto, pene, vulva, regione mediale delle cosce e parte inferiore dell'addome. Nei giovani adulti, dopo i trent'anni appaiono come papule circoscritte, solitarie o multiple, di colore rosso-scuro o nerastro, con una componente ipercheratosica poco marcata, possono associarsi a disturbi circolatori quali vene varicose, tromboflebite dello scroto e varicocele.

Gli angiocheratomi di Mibelli rappresentano un'affezione rara, a carattere autosomico dominante, caratterizzata da piccole formazioni angiomatose di colore rosso vivo, a superficie cheratosica, di aspetto talora verrucoso, disposti elettivamente sulle dita, sul dorso delle mani e dei piedi nonché nelle regioni mammarie. La malattia compare più frequentemente nell'adolescenza e si associa di solito all'acrocianosi e ai geloni.

L'angiocheratoma circoscritto neviforme si denota all'estremità con teleangectasie inizialmente solitarie, ma che diventano rapidamente multiple, tendono a divenire papu- 
lose e a raggrupparsi, estendendosi eccentricamente con aspetto serpiginoso e conformazione anulare per la tendenza all'atrofia centrale. Si manifesta durante l'infanzia, interessa di norma il sesso femminile e non presenta coinvolgimento sistemico.

Inoltre, è da ricordare che gli ANC si possono associare ad altre malattie di accumulo lisosomiale inclusa la malattia di Schindler, la GM1 gangliosidosi, l'aspartilglucosaminuria, sialidosi e la fucosidosi (11).

Da un punto di vista pratico nella valutazione dei pazienti con ANC multipli, l'ombelico deve sempre essere valutato dato che il suo interessamento è altamente evocativo di mAF. Inoltre, in letteratura vengono riportati rari casi di maschi adulti con poche lesioni, ma con storia personale o familiare particolarmente indicativa, comportando la necessità di escludere la malattia di Anderson-Fabry (7).

Il trattamento degli angiocheratomi è necessario quando sanguinano o per motivi estetici. Possono essere trattati mediante chirurgia convenzionale, diatermocoagulazione, crioterapia o con laserterapia. Quest'ultima rappresenta il trattamento golden standard per gli ANC, in quanto particolarmente efficace e offre i migliori risultati estetici. Diversi tipi di laser possono essere utilizzati quali argon laser, YAG laser, laser a luce pulsata e dye laser.

\section{Teleangectasie}

Le teleangectasie sono la seconda più comune manifestazione cutanea e si osservano, più frequentemente, nelle aree fotoesposte quali il viso e la regione sternale. Occasionalmente, soprattutto nei pazienti con angiocheratomi diffusi, si possono notare anche nelle sedi non fotoesposte. Sono state descritte teleangectasie delle labbra, del cavo orale e della lingua (3).

\section{Alterazioni della sudorazione}

I pazienti affetti da mAF lamentano frequentemente ipoidrosi o anidrosi provocati sia dal coinvolgimento delle terminazioni nervose autonomiche che dall'accumulo di Gb3 nelle ghiandole sudoripare eccrine e nei loro vasi sanguigni. Infatti negli assoni mielinici innervanti le ghiandole sudoripare eccrine sono state notate inclusioni lamellari; inclusioni citoplasmatiche e necrosi sono state osservate nelle cellule chiare della spirale secretoria. I piccoli vasi sanguigni attorno alle ghiandole sudoripare eccrine sono ristretti per la presenza di cellule endoteliali rigonfie con inclusioni pesanti. Questi depositi intracitoplasmatici potrebbero, quindi, essere responsabili della ridotta capacità di sudorazione nei pazienti con mAF con preponderante interessamento dei pazienti emizigoti (53\%-93\%). Per tale motivo i pazienti risultano intolleranti al calore, alle brusche variazioni della temperatura ambientale, all'esercizio fisico e possono accusare nausea, dispnea, cefalea e anche perdita di coscienza in ambienti caldi (12).

Raramente è stata riportata l'iperidrosi palmoplantare e della regione frontale con maggiore interessamento del genere femminile (13).

\section{Dismorfismo facciale}

I pazienti con assenza di attività enzimatica residua possono presentare dismorfismo facciale, anche se esso non rappresenta un segno clinico prominente. La faccia pseudo-acromegalica, chiamata "faccia Anderson-Fabry", si caratterizza per la fronte a incasso, creste sopraorbitarie prominenti, cisti a carico dei seni mascellari, prognatismo, labbra carnose, piramide nasale globosa con base ampia, padiglioni auricolari ispessiti e retroruotati (14).

\section{Manifestazioni orali e anomalie dentarie}

Più di un terzo dei pazienti sviluppa lesioni orali, infatti, oltre agli angiocheratomi e alle teleangectasie, è possibile ritrovare glossite, fissurazioni linguali e xerostomia (7).

Diversi studi riportano anomalie dentarie nei pazienti affetti da malattia di Anderson-Fabry. La malocclusione può essere un riscontro comune e si possono osservare anomalie dello sviluppo quali denti soprannumerari, agenesia, eruzione ritardata e discolorazione dentale generalizzata (3).

\section{Linfedema}

In alcuni pazienti affetti da mAF si osserva linfedema, specialmente pronunciato alle labbra, già descritto nei lavori originali di Anderson e Fabry, dovuto ad alterazioni strutturali e funzionali dei piccoli vasi linfatici della cute in seguito ad accumulo di glicosfingolipidi. Lo sviluppo del linfedema è accompagnato da obliterazione, frammentazione, ectasia e/o aumento della permeabilità dei vasi linfatici $(15,16)$.

\section{Fenomeno di Raynaud}

L'accumulo progressivo di Gb3 all'interno delle cellule vascolari comporta danni alla microcircolazione tali da promuovere il fenomeno Raynaud (13\%), un disturbo vasospastico caratterizzato dalla classica triade clinica di pallore intermittente (ischemia), cianosi e rubor (iperemia reattiva) delle dita di mani e piedi, delle orecchie e del naso in risposta a freddo, stress emotivo o altri eventi (17).

\section{Alterazione degli annessi piliferi}

Spesso può coesistere un rallentamento della crescita dei capelli e dei peli, in particolare agli arti inferiori, riconducibile a una carenza di nutrienti secondaria ad alterazioni della microcircolazione (7). 


\section{Conclusioni}

Le numerose manifestazioni dermatologiche rendono l'organo cute fondamentale per la diagnosi della mAF e la tempestiva istituzione della terapia enzimatica sostitutiva, che nell'ultimo decennio ha rivoluzionato la qualità e la possibilità di vita dei pazienti affetti da questa rara malattia $(18,19)$.

\section{Disclosures}

Financial support: No financial support was received for this submission.

Conflict of interest: The authors have no conflict of interest.

\section{Bibliografia}

1. Lidove O, Jaussaud R, Aractingi S. Dermatological and soft-tissue manifestations of Fabry disease: characteristics and response to enzyme replacement therapy. In: Mehta $A$, Beck $M$, SunderPlassmann G, eds. Fabry Disease: Perspectives from 5 Years of FOS. Oxford: Oxford PharmaGenesis; 2006. Chapter 24.

2. Orteu $\mathrm{CH}$, Jansen $\mathrm{T}$, Lidove $\mathrm{O}$, et al; FOS Investigators. Fabry disease and the skin: data from FOS, the Fabry outcome survey. Br J Dermatol. 2007;157(2):331-7.

3. Pistone G, Rizzo D, Bongiorno MR. Cutaneous complications of Anderson-Fabry disease. Curr Pharm Des. 2013,19(33):6031-6.

4. Larralde M, Luna PC. Fabry Disease. In: Wolff K, Goldsmith L, Kats S, Gilchrest B, Paller AS, Leffell D, eds. Fitzpatrick's Dermatology in General Medicine. 7th ed. New York: McGrawHill; 2008:1281-8.

5. Tokuriki A, Kiyohara T, Kumakiri M. Electron-microscopy of cherry haemangioma in the early diagnosis of Fabry disease. Acta Derm Venereol. 2013;93(4):471-2.

6. Larralde $\mathrm{M}$, Boggio $\mathrm{P}$, Amartino $\mathrm{H}$, Chamoles N. Fabry disease: a study of 6 hemizygous men and 5 heterozygous women with emphasis on dermatologic manifestations. Arch Dermatol. 2004; 140(12):1440-6.

7. Luna PC, Boggio P, Larralde M. Dermatologic Aspects of Fabry Disease. J Inborn Errors Metab Screen. 2016; 4:1-7.
8 Grazzini M, Stanganelli I, Rossari S, et al. Dermoscopy, confocal laser microscopy, and hi-tech evaluation of vascular skin lesions: diagnostic and therapeutic perspectives. Dermatol Ther. 2012;25(4):297-303.

9. Kanekura T, Fukushige T, Kanda A, et al. Immunoelectronmicroscopic detection of globotriaosylceramide accumulated in the skin of patients with Fabry disease. $\mathrm{Br} J$ Dermatol. 2005;153(3):544-8.

10. Zaballos P, Daufí C, Puig S, et al. Dermoscopy of solitary angiokeratomas: a morphological study. Arch Dermatol. 2007;143(3):318-25.

11. Kanitakis J, Allombert C, Doebelin B, et al. Fucosidosis with angiokeratoma. Immunohistochemical and electronmicroscopic study of a new case and literature review. J Cutan Pathol. 2005;32(7):506-11.

12. Jung SE, Kim YC. Hypohidrosis: an early clue in the diagnosis of Fabry disease. Clin Exp Dermatol. 2015;40(4):444-5.

13. Strutton DR, Kowalski JW, Glaser DA, Stang PE. US prevalence of hyperhidrosis and impact on individuals with axillary hyperhidrosis: results from a national survey. J Am Acad Dermatol. 2004;51(2):241-8.

14. Hogarth V, Hughes D, Orteu CH. Pseudoacromegalic facial features in Fabry disease. Clin Exp Dermatol. 2013;38(2): 137-9.

15. Jansen $\mathrm{T}$, Bechara FG, Orteu $\mathrm{CH}$, et al. The significance of lymphoedema in Fabry disease. Acta Paediatr Suppl. 2005; 447:117.

16. Amann-Vesti BR, Glitzelmann G, Widmer U, Bosshard NU, Steinmann B, Koppensteiner R. Severe lymphatic microangiopathy in Fabry disease. Lymphat Res Biol. 2003;1(3):185-9.

17. Germain DP, Atanasiu OI, Akrout-Marouene J, Benistan K. Raynaud's phenomenon associated with Fabry disease. J Inherit Metab Dis. 2015;38(2):367-8.

18. Wilcox WR, Banikazemi M, Guffon N, et al; International Fabry Disease Study Group. Long-term safety and efficacy of enzyme replacement therapy for Fabry disease. Am J Hum Genet. 2004;75(1):65-74.

19. Bongiorno MR, Pistone G, Aricò M. Fabry disease: enzyme replacement therapy. J Eur Acad Dermatol Venereol. 2003; 17(6):676-9. 